\title{
Minimize MIMO OFDM interference and noise ratio using polynomial-time algorithm
}

\author{
Muhamed K Husein \\ Department of Electrical Engineering, Tikrit University, Iraq
}

\begin{tabular}{|c|c|}
\hline Article Info & ABSTRACT \\
\hline Article history: & In the distributed transmit antenna MIMO OFDM system, each transmitting \\
\hline Received Apr 29, 2019 & and receiver due to the use of independent crystal oscillator. This paper \\
\hline Revised Jun 27, 2019 & proposes Polynomial-time algorithm for correcting the frequency offset in \\
\hline Accepted Sep 27, 2019 & $\begin{array}{l}\text { a received signal by maximizing the conditional average signal. The algorithm } \\
\text { focus on reducing to interference and noise ratio of each subcarrier on }\end{array}$ \\
\hline Keywords: & $\begin{array}{l}\text { the receiving antenna by frequency offset. The simulation result shows } \\
\text { the performance of the proposed algorithm is slightly improved compared with }\end{array}$ \\
\hline Algorithm & the existing frequency offset correction algorithm, and the complexity is \\
\hline BER & \\
\hline MIMO & \\
\hline OFDM & \\
\hline SNR & $\begin{array}{r}\text { Copyright } \odot 2020 \text { Institute of Advanced Engineering and Science. } \\
\text { All rights reserved. }\end{array}$ \\
\hline \multicolumn{2}{|l|}{ Corresponding Author: } \\
\hline \multicolumn{2}{|c|}{$\begin{array}{l}\text { Muhamed K Husein, } \\
\text { Department of Electrical Engineering, } \\
\text { Tikrit University, } \\
\text { Street of Tikrit-Mosil, Al-Qadissiyah quarter, 009642, Tikrīt, Sallahaldin, Iraq. } \\
\text { Email: muhamedmakhoodh@tu.edu.iq }\end{array}$} \\
\hline
\end{tabular}

\section{INTRODUCTION}

In the MIMO OFDM (Multi-Input Multi-Output Orthogonal Frequency Division Multiplexing) system [1-3], the difference between the local crystal oscillators of each distributed transmit antenna and the existence of multiple doppler shifts, causing different frequency offsets between each distributed transmit antenna to the receiver [4], inter-carrier interference (ICI) and system performance degradation. In recent years, this technology has also been gradually applied to MIMO OFDM systems mainly focus on antenna selection [5-8]. Antenna selection for MIMO-OFDM systems is based on subcarriers and subsystems. In order to combat multiple frequency offsets, the literature [9-11] adopts the equalization method to eliminate the frequency offset, but the performance of this method will degradate with increasing the offset frequency. In [4], a frequency offset correction algorithm for distributed transmit antenna MIMO OFDM is proposed, to improve the performance of the equalization-based frequency offset elimination method, which corrects multiple frequency offsets before equalization and reduces the interference caused by frequency offset [12-15]. The lower limit of the conditional average signal to interference and noise ratio (SINR, Signal-InterferenceNoise-Ratio) of the subcarriers on the antenna is the criterion correction frequency offset, but it has two disadvantages. the first, when the frequency deviation between the transmitting antennas increases, the lower limit of the conditional average SINR becomes more and more slack [4, 16, 17]. At this time, the frequency offset is corrected by maximizing the lower limit of the conditional average SINR. Conditional average SINR will produce a certain performance loss; the second, the algorithm needs to perform more trigonometric and inverse trigonometric operations, and the overall complexity is higher $[18,19]$.

In this paper a low complexity frequency offset correction algorithm proposed which directly correct the frequency offset by maximizing the conditional average SINR of subcarriers on each receiving antenna. In addition, analyses the receive antenna selection and signal processing algorithms based on MIMO-OFDM 
systems. By using the function in the conditional average SINR expression Polynomial approximation, deriving low-complexity frequency offset correction analytic. Compared with the literature [4], the performance of the proposed algorithm is slightly improved, and the complexity is reduced by $50 \%$ or more.

\section{MIMO-OFDM SYSTEM STRUCTURE AND CHANNEL MODEL}

When the subcarrier-based selection method is adopted, in the antenna subset selection process of each subcarrier, a single carrier MIMO system is actually faced, so the antenna selection method of the existing MIMO system can be introduced into the MIMO-Antenna selection in OFDM systems. Since the CSI is fed back to the transmitter when the transmit antenna is selected, this is not feasible when the channel changes randomly, so the receive antenna selection is more attractive. In theory, the choice of the receiving antenna will reduce the rank of the channel matrix, which will inevitably lead to a decrease in the channel capacity. If the corresponding signal combining processing algorithm is combined after the antenna selection, this performance loss can be compensated.

Figure 1 is a block diagram of the antenna selection at the receiving end of the MIMO-OFDM system. The signal received by the antenna selects an antenna to be demodulated by a specific antenna selection algorithm, and then recovers the information of the source through FFT, parallel string conversion, demodulation, and the like.

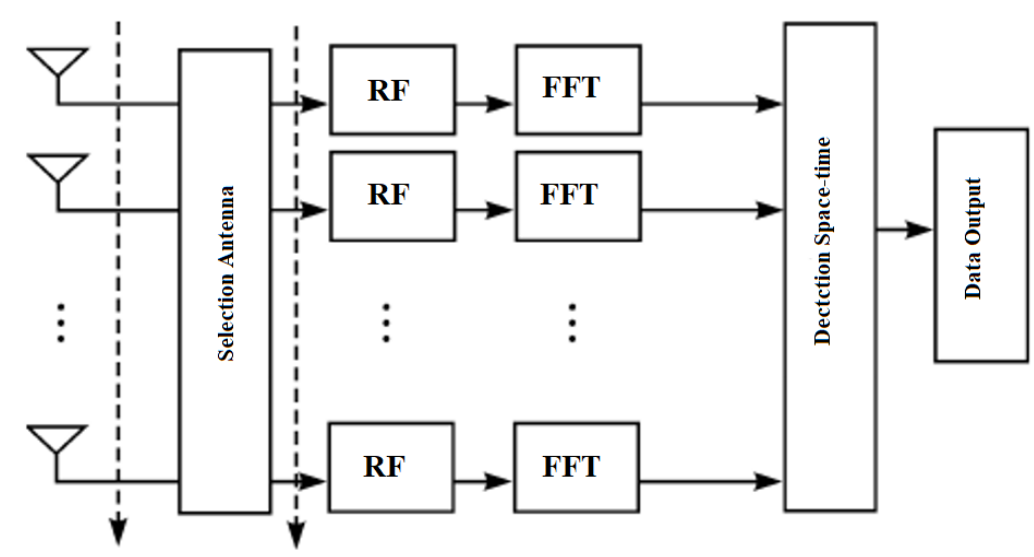

Figure 1. Block diagram of antenna selection at the receiving end of MIMO-OFDM system

\subsection{Transmitting signal}

Investigate a MIMO OFDM system using the MT root-distribution transmit antenna and the MR rootconcentrated receive antenna. Assume that the number of OFDM subcarriers is $K$, so $X_{m}^{k}(m=$ $\left.1,2, \ldots, M_{T} ; k=1,2, \ldots, K\right)$ that the information symbol carried by the $k t h$ subcarrier on the transmit antenna $\mathrm{m}$. K-point fast Fourier inverse after transforming and inserting the cyclic prefix (CP, Cyclic Prefix), at the discrete time 1 , the time domain signal on the transmitting antenna $\mathrm{m}$ is.

$$
S_{m}(l)=\frac{1}{\sqrt{K}} \sum_{k=0}^{K-1} X_{m}^{k} e^{j 2 \pi l / K} \quad-N_{g} \leq l \leq K-1
$$

Where $\mathrm{Ng}$ is the length of the $\mathrm{CP}$ and the $\mathrm{Ng}$ is equal to or greater than the sum of the maximum relative propagation delay between each transmit antenna and the maximum multipath delay of the channel [2].

\subsection{Frequency deviation model}

Due to the distribution of the transmitting antennas and the concentration of the receiving antennas, the distributed transmitting antennas use their respective local crystal oscillators, and the concentrated receiving antennas are used, a local oscillator [4]. Therefore, this paper assumes that the frequency offset between the same transmit antenna and the receiver antennas of the receiver is equal, and the frequency offset between different transmit antennas and the same receive antenna may be different. Let $\varepsilon_{\mathrm{m}}$ be the normalized frequency offset between the transmit antenna $m$ and the receiver antennas of the receiver (the ratio of the true frequency offset to the subcarrier spacing). Similar to the literature [4], this paper assumes that in the same OFDM symbol period, in the system. The difference between the maximum frequency offset and the minimum 
frequency offset is not more than half the subcarrier spacing, ie $\left\{\varepsilon_{m}\right\}-\min \left\{\varepsilon_{m}\right\} \leqslant \mathbf{0} .5$. Furthermore, assuming that $\varepsilon_{\mathrm{m}}$ remains constant within one OFDM symbol, it can randomly vary between OFDM symbols and can be accurately estimated by the receiver [20].

\subsection{Receiving signals}

It is assumed that the MIMO channel is spatially uncorrelated and has three characteristics of multipath Rayleigh fading, path loss, and shadow fading. Meanwhile, assuming that the channel remains unchanged within one OFDM symbol, the frequency of the $k$ th subcarrier on the receiving antenna $\mathrm{n}$ is assumed. The domain signal is:

$$
r_{n}^{k}=\sum_{m=1}^{M_{T}} \sqrt{P_{m}} H_{m}^{k} \Lambda_{m, n}^{0} X_{m}^{k}+\sum_{m=1}^{M_{r}} \sum_{q=0, q \neq k}^{k-1} \sqrt{P_{m}} H_{m}^{q} \Lambda_{m, n}^{q} X_{m}^{q}+w_{n}^{k}
$$

Where $H^{k}{ }_{m, n}$ is the frequency domain response of the small-scale multipath Rayleigh fading on the subcarrier $\mathrm{k}$ between the transmitting antenna $\mathrm{m}$ and the receiving antenna $\mathrm{n}$, and $\mathrm{n}$ is a zero-mean complex Gaussian random variable with $H_{m, n}^{k}$ a variance of $1 ; P_{m}$ is the transmitting antenna $\mathrm{m}$ to the receiving The large-scale fading coefficient between machines, which characterizes path loss and shadow fading;

$$
\Lambda_{m, n}^{k}=\frac{\sin \left(\pi\left(k+\varepsilon_{m}\right)\right)}{K \sin \left(\pi\left(k+\varepsilon_{m}\right) / K\right)} e^{\left.j \tau\left(K-\frac{1}{K}\right)\left(k+\varepsilon_{m}\right)\right)}
$$

is the ICI coefficient [21];

$$
D_{m}^{k}=\sum_{m=1}^{M_{T}} \sqrt{P_{m}} H_{m}^{k} \Lambda_{m, n}^{0} X_{m}^{k}
$$

is the effective data item;

$$
I_{n}^{k}=\sum_{m=1}^{M_{r}} \sum_{q=0, q \neq k}^{k-1} \sqrt{P_{m}} H_{m}^{q} \Lambda_{m, n}^{q} X_{m}^{q}
$$

is the ICI interference; $w_{m}^{k}$ is the zero-mean complex Gaussian white noise with $\sigma^{2}{ }_{w}$ variance.

\section{PROPOSED METHOD}

The receiver multiplies the received signal of each receiving antenna by $\exp \left(-\frac{j 2 \pi \tilde{\varepsilon}_{n} I}{K}\right)$ the frequency offset correction signal in the time domain to correct the frequency offset, where $\tilde{\varepsilon}_{n}$ is the normalized frequency offset correction value on the receiving antenna $\mathrm{n}$ [4]. The proposed frequency offset correction algorithm for maximizing the lower bound of the conditional average $\operatorname{SINR}\left(P_{m}, \varepsilon_{m}\right.$ and $\tilde{\varepsilon}_{n}$ based on the conditional average SINR) of the subcarriers on each receiving antenna, this paper directly maximizes the conditional average SINR of the subcarriers on each receiving antenna. For the target, after correcting the frequency offset and frequency offset correction, the frequency domain signal of the kth subcarrier on the receiving antenna $n$ is:

$$
r_{n}^{k}\left(\tilde{\varepsilon}_{n}\right)=D_{n}^{k}\left(\tilde{\varepsilon}_{n}\right)+I_{n}^{k}\left(\tilde{\varepsilon}_{n}\right)+w_{n}^{k}\left(\tilde{\varepsilon}_{n}\right)
$$

where

$$
D_{m}^{k}\left(\tilde{\varepsilon}_{n}\right)=\sum_{m=1}^{M_{T}} \sqrt{P_{m}} H_{m}^{k} \Lambda_{m, n}^{0}\left(\tilde{\varepsilon}_{n}\right) X_{m}^{k}
$$

and

$$
I_{n}^{k}\left(\tilde{\varepsilon}_{n}\right)=\sum_{m=1}^{M_{r}} \sum_{q=0, q \neq k}^{k-1} \sqrt{P_{m}} H_{m}^{q} \Lambda_{m, n}^{q}\left(\tilde{\varepsilon}_{n}\right) X_{m}^{q}
$$

is the effective signal and ICI interference after the frequency offset correction; 


$$
\Lambda_{m, n}^{k}\left(\tilde{\varepsilon}_{n}\right)=\frac{\sin \left(\pi\left(k+\varepsilon_{m}-\tilde{\varepsilon}_{n}\right)\right)}{K \sin \left(\pi\left(k+\varepsilon_{m}-\tilde{\varepsilon}_{n}\right) / K\right)}
$$

is the zero mean complex Gaussian white noise $w_{m}^{k}$ with $\sigma^{2}{ }_{w}$ variance.

Assuming $X_{m}^{k}$ that the zero mean is independent and identically distributed random variable with variance of $1, I_{n}^{k}\left(\tilde{\varepsilon}_{n}\right)$ the conditional mean and conditional variance are [4]:

$$
\begin{aligned}
& E\left[I_{n}^{k}\left(\tilde{\varepsilon}_{n}\right) \mid P_{m}, \varepsilon_{m}, \tilde{\varepsilon}_{n}\right]=0 \\
& E\left[I_{n}^{k}\left(\tilde{\varepsilon}_{n}\right) \mid P_{m}, \varepsilon_{m}, \tilde{\varepsilon}_{n}\right]=\sum_{m=1}^{M_{r}} P_{m}\left(1-\left(\frac{\sin \left(\pi\left(k+\varepsilon_{m}-\tilde{\varepsilon}_{n}\right)\right)}{K \sin \left(\pi\left(k+\varepsilon_{m}-\tilde{\varepsilon}_{n}\right) / K\right)}\right)^{2}\right)
\end{aligned}
$$

Thus, the $k_{t h}$ subcarrier on the receiving antenna $\mathrm{n}$ is the conditional average SINR of:

$$
\gamma_{n}^{k}\left(\tilde{\varepsilon}_{n}\right)=\frac{E\left[\left.D_{n}^{k}\left(\tilde{\varepsilon}_{n}\right)\right|^{2} \mid P_{m}, \varepsilon_{m}, \tilde{\varepsilon}_{0}\right]}{E\left[\left.I_{n}^{k}\left(\tilde{\varepsilon}_{n}\right)\right|^{2} \mid P_{m}, \varepsilon_{m}, \tilde{\varepsilon}_{0}\right]}=\frac{\sum_{m=1}^{2} P_{m} \operatorname{sinc} c^{2}\left(\varepsilon_{n}-\tilde{\varepsilon}_{n}\right)}{\sum_{m=1}^{2} P_{m}\left(1-\operatorname{sinc}^{2}\left(\varepsilon_{n}-\tilde{\varepsilon}_{n}\right)\right)+\sigma_{w}^{2}}
$$

Since the number of subcarriers K is much larger than $\left(\varepsilon_{m}-\tilde{\varepsilon}_{n}\right)$, so $k \sin \left(\frac{\pi\left(\varepsilon_{m}-\tilde{\varepsilon}_{n}\right)}{K}\right) \approx \pi\left(\varepsilon_{m}-\tilde{\varepsilon}_{n}\right)$ [22], then (6) can be reduced to:

$$
\gamma_{n}^{k}\left(\tilde{\varepsilon}_{n}\right)=\frac{\sum_{m=1}^{M_{r}} P_{m} \operatorname{sinc}^{2}\left(\varepsilon_{n}-\tilde{\varepsilon}_{n}\right)}{\sum_{m=1}^{M_{r}} P_{m}\left(1-\operatorname{sinc}^{2}\left(\varepsilon_{n}-\tilde{\varepsilon}_{n}\right)\right)+\sigma_{w}^{2}}
$$

Among them

$$
\operatorname{sinc}(x)=\left\{\begin{array}{cc}
\frac{\sin (\pi x)}{\pi x} & x \neq 0 \\
1 & x=0
\end{array}\right.
$$

From (7) that, $\gamma_{n}^{k}\left(\tilde{\varepsilon}_{n}\right)$ regardless of the subcarrier label $k$, the conditional average SINR of each subcarrier on the same receiving antenna is equal. For convenience, let:

$$
\gamma_{n}\left(\tilde{\varepsilon}_{n}\right)=\gamma_{n}^{k}\left(\tilde{\varepsilon}_{n}\right)
$$

Thus, $n$ the optimal frequency offset correction value that maximizes the conditional average SINR of each subcarrier on the receiving antenna $\tilde{\varepsilon}_{n, o p t}$ can be expressed as:

$$
\tilde{\varepsilon}_{n, o p t}=\operatorname{argmax}_{\tilde{\varepsilon}_{n}} \gamma_{n}\left(\tilde{\varepsilon}_{n}\right) \quad n=1, \ldots, M_{R}
$$

Since $\gamma_{n}^{k}\left(\tilde{\varepsilon}_{n}\right)$ about incrementing $\sum_{m=1}^{M_{T}} P_{m} \operatorname{sinc}^{2}\left(\varepsilon_{m}-\tilde{\varepsilon}_{n}\right),(10)$ can be simplified to:

$$
\tilde{\varepsilon}_{n, o p t}=\sum_{m=1}^{M_{T}} P_{m} \operatorname{sinc}^{2}\left(\varepsilon_{m}-\tilde{\varepsilon}_{n}\right)
$$

From (11), $\tilde{\varepsilon}_{n, o p t}$ it is difficult to solve the problem directly. The approximate polynomial of the function $\operatorname{Sinc}^{2}(x)$ is used to solve the problem.

Select the approximate polynomial as a quadratic polynomial, using Lagrangian interpolation [23], then $\operatorname{sinc}^{2}(\mathrm{x})$ in the interval $[-0.5,0.5]$ can be approximated as:

$$
\operatorname{sinc}^{2}(x) \approx \sum_{j=0}^{2} \operatorname{sinc}^{2}\left(x_{j}\right) Q_{j(x)} \quad-0.5 \leq x \leq 0.5
$$

Among them,

$$
Q_{j(x)} \prod_{t=0, t \neq j}^{2} \frac{\left(x-x_{t}\right)}{\left(x_{j}-x_{t}\right)} \quad x_{j}=\frac{1}{2} \cos \left(\frac{(2 j+1) \pi}{6}\right) \quad j=0,1,2
$$


After the operation, the (12) can be written as:

$$
\operatorname{sinc}^{2}(x) \approx 1-a x^{2} \quad-0.5 \leq x \leq 0.5
$$

Where $\mathrm{a}=2.5771$.

Substituting (13) into (11), there are:

$$
\tilde{\varepsilon}_{n, o p t}=\operatorname{argmax}_{\tilde{\varepsilon}_{n}} \sum_{m=1}^{M_{T}} P_{m} \operatorname{sinc}^{2}\left(\varepsilon_{m}-\tilde{\varepsilon}_{n}\right)=\operatorname{argmax}_{\tilde{\varepsilon}_{n}} f\left(\tilde{\varepsilon}_{n}\right)
$$

Where $f\left(\tilde{\varepsilon}_{n}\right)=\sum_{m=1}^{M_{T}} P_{m}\left(1-a\left(\varepsilon_{m}-\tilde{\varepsilon}_{n}\right)^{2}\right)$. The first derivative of $f\left(\tilde{\varepsilon}_{n}\right)$ the order is equal to zero, the stagnation point of $f\left(\varepsilon_{n}\right)$ can be found as:

$$
\tilde{\varepsilon}_{n, 0}=\sum_{m=1}^{M_{T}} P_{m} \varepsilon_{m} / \sum_{m=1}^{M_{T}} P_{m}
$$

Substituting $\tilde{\varepsilon}_{n, 0}$ into of $f\left(\tilde{\varepsilon}_{n}\right)$, the second derivative with:

$$
\frac{d^{2} f\left(\tilde{\varepsilon}_{n}\right)}{d \tilde{\varepsilon}_{n}^{2}}=-2 z \sum_{m=1}^{M_{r}} P_{m}<0
$$

Therefore, $f\left(\tilde{\varepsilon}_{n}\right)$ reach the maximum value at $\tilde{\varepsilon}_{n}=\tilde{\varepsilon}_{n, 0}$.

$$
\tilde{\varepsilon}_{n, o p t} \approx \tilde{\varepsilon}_{n, 0}=\sum_{m=1}^{M_{T}} P_{m} \varepsilon_{m} / \sum_{m=1}^{M_{T}} P_{m}
$$

From (17), it is known that $\tilde{\varepsilon}_{n, o p t}$ is independent of the receiving antenna number $n$, so the optimal frequency offset correction value on each receiving antenna is the same, that is,

$$
\tilde{\varepsilon}_{1, o p t}=\tilde{\varepsilon}_{2, o p t} \ldots=\tilde{\varepsilon}_{n, o p t}=\tilde{\varepsilon}_{M_{g}, o p t} \approx \sum_{m=1}^{M_{T}} P_{m} \varepsilon_{m} / \sum_{m=1}^{M_{T}} P_{m}
$$

\section{RESULT AND DISCUSSION}

The simulation parameters as follows: 2 transmit 2 receive, QPSK modulation, information symbols $X_{m}^{k}$ are independent of each other; OFDM subcarrier number is $K=128, \mathrm{CP}$ length is $\mathrm{Ng}=32$, subcarrier spacing is $20 \mathrm{kHz}$; small scale fading between pairs of transceiver antennas The channels are independent of each other and are modelled as a two-path gain-Rayleigh channel [24] with a two-path spacing of 392.623ns; the normalized frequency offset $\varepsilon_{\mathrm{m}}$ obeys a uniform distribution (ie, $\varepsilon_{m} \sim U[a, b], a, b$ the values are given below), and $\varepsilon_{m}$ and $\varepsilon_{\widetilde{m}}$ are independent of each other at $m \neq \widetilde{m}$; the large-scale fading coefficient Pm remains constant during the simulation, and the receiver adopts zero-forcing detection.

Figure 2 shows the case where the symbol error rate varies with the frequency offset correction value $\varepsilon_{2}$ of the receiving antenna 2 in two cases, where SIR $=30 \mathrm{~dB},[-0.2,0.2]$. In case 1 , the frequency offset correction value of the receiving antenna 1 is fixed as the sum point, where, case 2 is the optimal frequency offset correction value, $p=1, \ldots, 5$. Fig. 1 shows that the error symbol rate in both scenarios is the smallest at the time, which indicates that the numerical results are consistent with the simulation results.

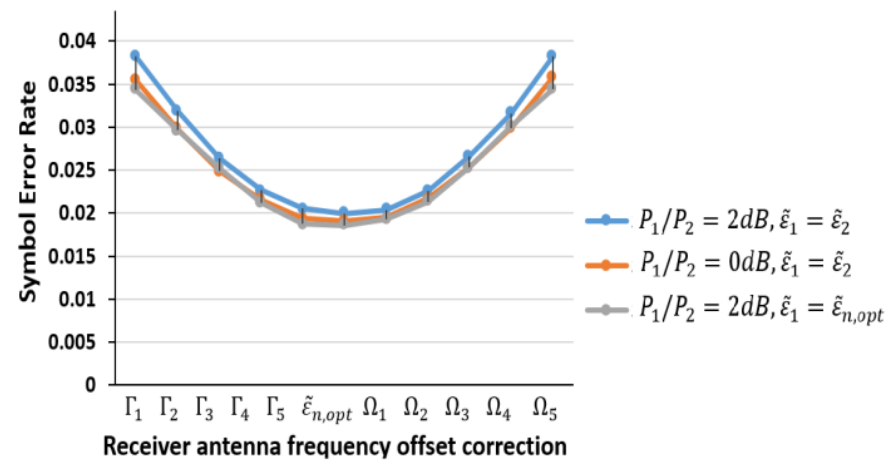

Figure 2. The relationship between symbol error rate and frequency corrections received by antenna 2 
Figure 3 shows the comparison of the sub-carrier theoretical conditional average signal-tointerference ratio (SIR) after using the proposed algorithm, to correct the frequency offset (11). Here, the theoretical conditional average SIR is defined as:

$$
S I R=\frac{E\left[\left.D_{n}^{k}\left(\tilde{\varepsilon}_{n}\right)\right|^{2} \mid P_{m}, \varepsilon_{m}, \tilde{\varepsilon}_{0}\right]}{E\left[\left.I_{n}^{k}\left(\tilde{\varepsilon}_{n}\right)\right|^{2} \mid P_{m}, \varepsilon_{m}, \tilde{\varepsilon}_{0}\right]}=\frac{\sum_{m=1}^{2} P_{m} \operatorname{sinc}^{2}\left(\varepsilon_{n}-\tilde{\varepsilon}_{n}\right)}{\sum_{m=1}^{2} P_{m}\left(1-\operatorname{sinc}^{2}\left(\varepsilon_{n}-\tilde{\varepsilon}_{n}\right)\right)}
$$

Where, $\tilde{\varepsilon}_{n}$ is value of the frequency offset correction.

Figure 3 shows that the SIR curve of the proposed algorithm agrees well with the ideal algorithm, while the SIR curve of the existing algorithm is gradually lower than $\varepsilon_{2}-\varepsilon_{1}$ the ideal algorithm as the frequency deviation between the two transmitting antennas increases. This is because with the increase, the condition $\varepsilon_{2}-\varepsilon_{1}$ lower limit of the average SIR will be more and more relaxed [4]. At this time, correcting the frequency offset (the existing algorithm) with the criterion of maximizing the conditional average SIR will bring more obvious errors.

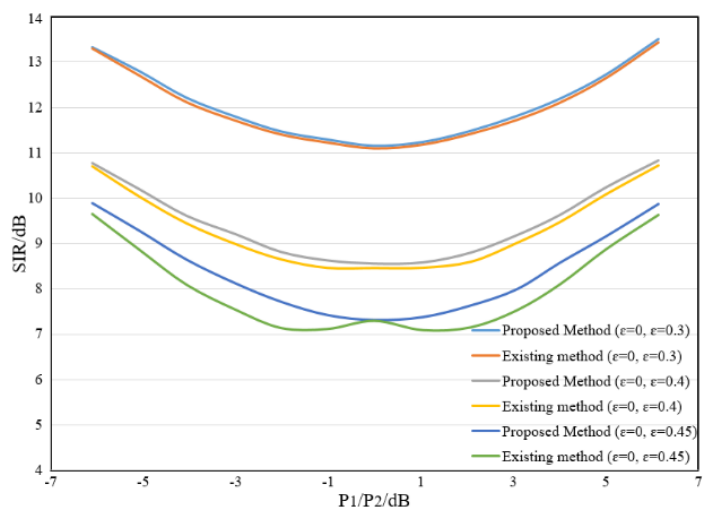

Figure3. The subcarriers after correcting frequency offset

Figure 4 compares the error rate of the proposed algorithm with the existing algorithm, where $\mathrm{P}_{1} / \mathrm{P}_{2}=3 \mathrm{~dB}, \varepsilon_{m} \sim U[-0.24,0.24]$. Figure 4 shows the error sign after frequency offset correction. The performance of the rate is better than that of the uncorrected frequency offset, and the performance of the proposed algorithm is slightly better than the existing algorithm.

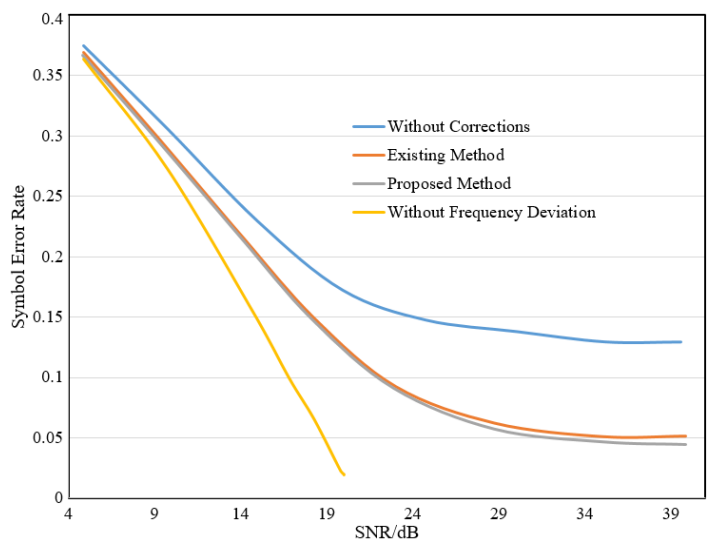

Figure 4. Comparison of symbol error rates

The complexity analysis is carried out, and the complexity of this algorithm is compared with the existing algorithm (i.e, [4]). The frequency offset correction value of each existing antenna on the receiving antenna is [4]: 


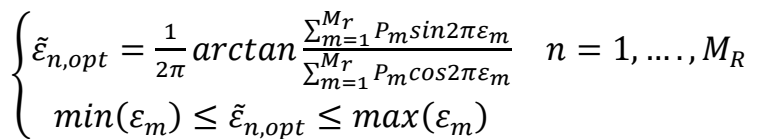

This paper validate the complexity of the proposed algorithm based on [25]. According to (17) and (20), Table 1 lists the computational complexity of the proposed algorithm and the existing algorithm.

Table 1 Comparison of the complexity with the existing algorithm [3]

\begin{tabular}{cccccccc}
\hline & Addition & Multiplication & Division & Sine & Cosine & Constant & Total number of operations \\
\hline Existing algorithm [3] & $2\left(M_{T}-1\right)$ & $2 M_{T}$ & 2 & $M_{T}$ & $M_{T}$ & 1 & $6 M_{T}+1$ \\
Proposed algorithm & $2\left(M_{T}-1\right)$ & $M_{T}$ & 1 & 0 & 0 & 0 & $3 M_{T}-1$ \\
\hline
\end{tabular}

It can be seen from Table 1 that the total number of operations of the algorithm is $3 \mathrm{M}_{\mathrm{T}}-1$ times, and the existing algorithm is $6 \mathrm{M}_{\mathrm{T}}+1$ times. The algorithm of this paper is lower than the existing algorithm. At the same time, for any operation required to complete the algorithm, the algorithm needs the number of times is less than or equal to the existing algorithm. In addition, the existing algorithm requires $\mathrm{M}_{\mathrm{T}}$ sine operation, $\mathrm{M}_{\mathrm{T}}$ cosine operation and 1 arctangent operation. Defined $C\left(M_{T}\right)=\left(3 M_{T}-1\right) /\left(6 M_{T}+1\right)$ as the complexity ratio function of the proposed algorithm and the existing algorithm. Because of the first derivative of $\frac{d C\left(M_{T}\right)}{d M_{T}}=9 /\left(6 M_{T}+1\right)^{2}>0, \mathrm{C}\left(\mathrm{M}_{\mathrm{T}}\right)$ is increasing with respect to the number of transmitting antennas $\mathrm{M}_{\mathrm{T}}$ tends to infinity. In terms of the number of operations $\lim _{M_{T} \rightarrow \infty} C\left(M_{T}\right)=0.5$, so the proposed method complexity is less than or equal to $50 \%$ of the existing algorithm.

\section{CONCLUSION}

In this paper, a multi-frequency offset correction algorithm for distributed transmit antenna MIMO OFDM is proposed. The conditional average SIR is maximized for each subcarrier on the receive antenna as the criterion to correct the frequency offset, by the function $\operatorname{Sin} c^{2}(*)$ in the conditional average SINR expression. The polynomial approximate method is obtained with low complexity frequency offset correction. Compared with the existing frequency offset correction algorithm, the performance of the proposed algorithm is slightly improved, and the complexity is reduced by $50 \%$ or more.

\section{ACKNOWLEDGEMENTS}

The authors are grateful to Tikrit University for providing financial support to complete this project.

\section{REFERENCES}

[1] N. H. Dawod, I. D. Marsland, and R. H. Hafez, "Improved transmit null steering for MIMO-OFDM downlinks with distributed base station antenna arrays," IEEE Journal on Selected Areas in Communications, vol. 24, pp. 419-426, 2006.

[2] Y. Shen, Y. Tang, T. Kong, and S. Shao., "Optimal antenna location for STBC-OFDM downlink with distributed transmit antennas in linear cells," IEEE Communications Letters, vol. 11, pp. 387-389, 2007.

[3] P. A. C. Lopes and J. A. B. Gerald, "Leakage-based precoding algorithms for multiple streams per terminal MUMIMO systems," Digital Signal Processing, vol. 75, pp. 38-44, 2018.

[4] K. Deng, Y. Tang, S. Shao, and K. Sun., "Correction of carrier frequency offsets in OFDM-based spatial multiplexing MIMO with distributed transmit antennas," IEEE Transactions on Vehicular Technology, vol. 58, pp. 2072-2077, 2009.

[5] B.-b. Hu, Y.-a. Liu, G. Xie, J.-c. Gao, and Y.-1. Yang., "Energy efficiency of massive MIMO wireless communication systems with antenna selection," The Journal of China Universities of Posts and Telecommunications, vol. 21, pp. 1-8, 2014.

[6] E. M. Okumu and M. E. Dlodlo, "Transmit antenna selection for multiple antenna systems with stall avoidance," Computers \& Electrical Engineering, vol. 58, pp. 144-153, 2017.

[7] A. Datta and V. Bhatia, "A near maximum likelihood performance modified firefly algorithm for large MIMO detection," Swarm and Evolutionary Computation, vol. 44, pp. 828-839, 2019.

[8] O. Font-Bach, N. Bartzoudis, A. Pascual-Iserte, and D. L. Bueno., "A real-time MIMO-OFDM mobile WiMAX receiver: Architecture, design and FPGA implementation," Computer Networks, vol. 55, pp. 3634-3647, 2011.

[9] A. Trimeche, A. Sakly, and A. Mtibaa., "FPGA Implementation of ML, ZF and MMSE Equalizers for MIMO Systems," Procedia Computer Science, vol. 73, pp. 226-233, 2015. 
[10] A. Kumar and P. R. Sahu, "Performance analysis of DCSK-SR systems based on best relay selection in multiple MIMO relay environment," AEU - International Journal of Electronics and Communications, vol. 70, pp. 18-24, 2016.

[11] W. Zhang, D. Qu, and G. Zhu., "Performance investigation of distributed STBC-OFDM system with multiple carrier frequency offsets," in 2006 IEEE 17th International Symposium on Personal, Indoor and Mobile Radio Communications, pp. 1-5, 2006.

[12] Lindner, J., et al., Comparison of vector detection algorithms for MIMO-OFDM. AEU - International Journal of Electronics and Communications, 2005. 59(3): p. 137-146.

[13] Upadhya, K., C.S. Seelamantula, and K.V.S. Hari, A risk minimization framework for channel estimation in OFDM systems. Signal Processing, 2016. 128: p. 78-87.

[14] Golovins, E. and N. Ventura, Optimisation of the pilot-to-data power ratio in the wireless MIMO-OFDM system with low-complexity MMSE channel estimation. Computer Communications, 2009. 32(3): p. 465-476.

[15] Chinnadurai, S., et al., Worst-case weighted sum-rate maximization in multicell massive MIMO downlink system for 5G communications. Physical Communication, 2018. 27: p. 116-124.

[16] C. Papathanasiou, N. Dimitriou, and L. Tassiulas, "Dynamic radio resource and interference management for MIMOOFDMA mobile broadband wireless access systems," Computer Networks, vol. 57, pp. 3-16, 2013.

[17] H. Tao, M. Z. A. Bhuiyan, A. N. Abdalla, M. M. Hassan, J. M. Zain, and T. Hayajneh., "Secured data collection with hardware-based ciphers for iot-based healthcare," IEEE Internet of Things Journal, vol. 6, pp. 410-420, 2019.

[18] Y. Wang and X.-f. Tao, "Inter-antenna and subblock shifting and inversion for peak-to-average power ratio reduction in MIMO-OFDM systems," The Journal of China Universities of Posts and Telecommunications, vol. 14, pp. 41-45, 2007.

[19] S. Singh and A. Kumar, "Performance Analysis of Adaptive Clipping Technique for Reduction of PAPR in Alamouti Coded MIMO-OFDM Systems," Procedia Computer Science, vol. 93, pp. 609-616, 2016.

[20] A. S. Namitha and S. M. Sameer, "A bandwidth efficient selective mapping technique for the PAPR reduction in spatial multiplexing MIMO-OFDM wireless communication system," Physical Communication, vol. 25, pp. 128-138, 2017.

[21] P. Remlein, T. Çogalan, and T. Gucluoglu., "OFDM with transmit and receive antenna selection based on subcarrier groups," in 2012 8th International Symposium on Communication Systems, Networks \& Digital Signal Processing (CSNDSP), pp. 1-4, 2012.

[22] D. N. Dao and C. Tellambura, "Intercarrier interference self-cancellation space-frequency codes for MIMO-OFDM," IEEE Transactions on Vehicular Technology, vol. 54, pp. 1729-1738, 2005.

[23] S. S. Ray., Numerical analysis with algorithms and programming: CRC Press, 2018.

[24] D. Huang and K. B. Letaief, "An interference-cancellation scheme for carrier frequency offsets correction in OFDMA systems," IEEE Transactions on Communications, vol. 53, pp. 1155-1165, 2005.

[25] S. Marinkovic, B. Vucetic, and A. Ushirokawa, "Space-time iterative and multistage receiver structures for CDMA mobile communication systems," IEEE Journal on selected Areas in Communications, vol. 19, pp. 1594-1604, 2001.

\section{BIOGRAPHY OF AUTHOR}

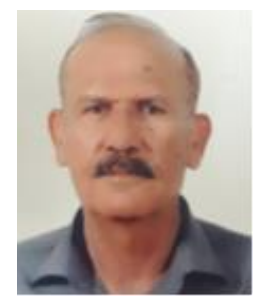

Mohamed Kh. Husein, He got his B.Sc Electronics Engineering, Air-Military Engineering Academy/Faculty of Electronics. /Sarajevo Yugoslavia, 1982. Also he got his M.Sc. Electronics Engineering, University of Belgrade / Electrical Engineering College, Belgrade Yugoslavia. 1984. Currently, working with college of engineering Shirqat, department of electrical engineering, Tikrit university. His main research interest including, wireless communication, OFDM, and optimization. 